\title{
Evidence for ultra-cold traps and surface water ice in the lunar south polar crater
} Amundsen

E. Sefton-Nash $^{\mathrm{a}, *}$, J.-P. Williams ${ }^{\mathrm{b}}$, B. T. Greenhagen ${ }^{\mathrm{c}}$, T. J. Warren ${ }^{\mathrm{d}}$, J. L. ap afield ${ }^{\mathrm{e}}$, K.-M. Aye ${ }^{\mathrm{f}}$, F. Leader ${ }^{\mathrm{g}}$, M. A. Siegler ${ }^{\text {, P. O. Hayne }}{ }^{i}$, N. Bowles ${ }^{\text {d, D. A. Paige }}{ }^{b}$

${ }^{a}$ European Space Research and Technology Center (ESTEC), European Space Agency, 2200 AG Noordwijk, the Netherlan

${ }^{\mathrm{b}}$ Department of Earth, Planetary and Space Sciences, University of California Los Angeles, 595 Charles Young Drive East, BD

c Johns Hopkins University Atmospheric Physics Laboratory, 11101 Johns Hopkins Road, Laurel, MD 20723, USA

${ }^{\mathrm{d}}$ Atmospheric, Oceanic and Planetary Physics, University of Oxford, Department of Physics, Clarendon Laboratory, Parks Road, Oxford OX1 3PU, United Kingdom

${ }^{\text {e }}$ Space Science Institute, 4750 Walnut Street, Suite 205, Boulder, CO 80301, USA

${ }^{\mathrm{f}}$ Laboratory for Atmospheric and Space Physics (LASP), 3665 Discovery Drive, Boulder, CO 80303, USA

${ }^{g}$ Jet Propulsion Laboratory, California Institute of Technology, Pasadena, CA 91109, USA

${ }^{\mathrm{h}}$ Planetary Science Institute, 1700 East Fort Lowell, Suite 106, Tucson, AZ, USA

${ }^{\mathrm{i}}$ Astrophysical \& Planetary Sciences, University of Colorado Boulder, 391 UCB, 2000 Colorado Ave,

\section{A R T I C L E I N F O}

\section{A B S T R A C T}

\section{Keywords:}

Moon

Permanent shadow

Polar

Volatiles

Emissivity

The northern floor and wall of And disen crater, near the lunar south pole, is a permanently shaded region (PSR). Previous study aris area using data from the Lunar Orbiter Laser Altimeter (LOLA), Diviner and LAMP instruments aboard anar Reco naissance Orbiter (LRO) shows a spatial correlation between brighter $1064 \mathrm{~nm}$ albedo, annual ma mum sur ce temperatures low enough to enable persistence of surface water ice $(<110 \mathrm{~K})$, and noma ult siolet radiation. We present results using data from Diviner that quantify the differential en osivitios observed in the far-IR (near the Planck peak for PSR-relevant temperatures) between the PSR and neary non SR target in Amundsen Crater.

We find fear s in f. IR emissivity $(50-400 \mu \mathrm{m})$ could be attributed to either, or a combination, of two effects (i) ontian omissive behavior between permanently-shadowed temperature regimes and those of nor ally i inated polar terrain, perhaps related to presence of water frost (as indicated in other studies), or (i high egrees-of anisothermality within observation fields of view caused by doubly-shaded areas within the PSR ret that are colder than observed brightness temperatures.

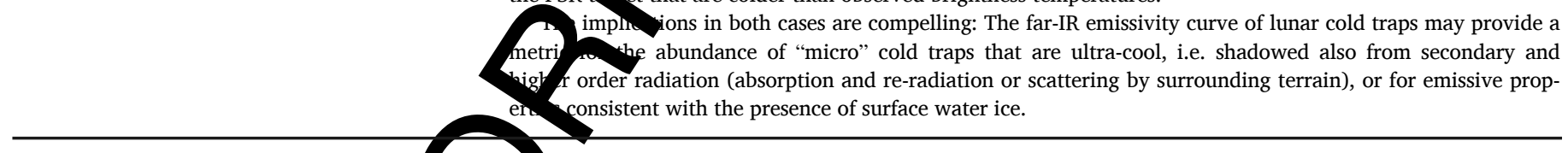

\section{Introduction}

Permanently shadowed regio $S$ (PSRs) to the Moon's low axial tilt. Crat interio pressions act as cold traps anc nay surface or subs face volatiles. The predominant sources of radiation in are immted to upwelling heat flow from the lunar interior, stek radion, secondary illumination by reflected

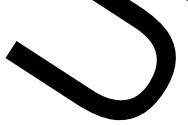

\footnotetext{
* Corresponding author.

Email address: e.sefton-nash@cosmos.esa.int (E. Sefton-Nash)
}

https://doi.org/10.1016/j.icarus.2019.06.002

Received 2 October 2018; Received in revised form 17 May 2019; Accepted 3 June 2019

Available online $\mathrm{xxx}$

0019-1035/ (c) 2018. or scattered light from illuminated surfaces, or thermal emission from nearby warmer shadowed surfaces. These radiative sources are often negligible, allowing PSRs to remain at very low temperatures that are more akin to those observed on bodies in the outer solar system.

Recent studies of the lunar poles using data from NASA's Lunar Reconnaissance Orbiter (LRO) have focussed on isolating potential signatures of such volatiles, following the detection by the Lunar Crater Observation and Sensing Satellite (LCROSS) (Schultz et al., 2010) of water 
and other volatiles in a plume of material ejected from Cabeus crater by the impact of a spent rocket stage (Colaprete et al., 2010). The body of results to date does not conclusively resolve the distribution of volatile abundance at the surface, nor in near-surface regolith layers. However, work to determine the presence of surface volatiles in the lunar South Polar region is generally consistent with laterally discontinuous veneers of water ice (Hayne et al., 2014, 2015; Fisher et al., 2017, Li et al., 2018).

We analyse far-IR data from the South Polar Amundsen crater to explore the differences in far-IR emission, as observed by the Diviner Lunar Radiometer Experiment aboard LRO (Paige et al., 2010a). We select a partially illuminated target as a control (i.e. where we would not expect to find evidence for surface volatiles due to the high daytime temperatures), and a permanently shadowed target identified as a high priority site for study of lunar volatiles (Lemelin et al., 2014), and in which there is evidence in remote sensing data for surface water ice (Hayne et al., 2015; Fisher et al., 2017).

\section{Targets in Amundsen crater}

Compared to other major south polar PSRs, the PSR in Amundsen crater offers the advantage of being predominantly outside the reduced observation coverage in polar regions. This is caused by a combination of a reduction in LRO's orbit inclination 2009-2016, and the nominal nadir pointing of Diviner.

We define target boundaries at a $3 \mathrm{~km}$ radius around centers of $93.1047^{\circ} \mathrm{E}, 84.5523^{\circ} \mathrm{S}$ and $91.2826^{\circ} \mathrm{E}, 83.6889^{\circ} \mathrm{S}$ for non-PSR (NPSR) (i.e. partially illuminated) and permanently shadowed (PSR) targets, respectively (Fig. 1). For the PSR target this radius ensures that only observations from within the PSR boundary are included, but the size is also a balance between minimizing area (to minimize scatter cause by heterogeneous thermal states) and ensuring sufficient data cover for robust statistics, and computational capabilities.

Our permanently shadowed target includes terrain that is ref rted as having both surface temperatures and local slopes low enoy h to able persistence of surface water ice $\left(<110 \mathrm{~K}\right.$ and $<10^{\circ}$ and brighter $1064 \mathrm{~nm}$ albedo, as measured by Lunar Orb timeter (LOLA) reflectance (Fisher et al., 2017). However, manently shadowed areas, and only a portion of our this correlation between bright $1064 \mathrm{~nm}$ albedo an

A similar correlation was performed by Hayne tween annual maximum temperature and anomen ult iolet radiation as measured by the Lyman Alpha $\mathrm{V}$, ppin woject (LAMP) (Gladstone et al., 2010). In that study, UV ectr of Psks were obtained by measuring reflectance of starlight ano mission from interplanetary hydrogen. The spectral featur or mer-10 in the UV were characterized by LAMP by observing (i) decre sing albedo in the $129.57-155.57 \mathrm{~nm}$ bandpass, (ii) ncreasin albedo in the

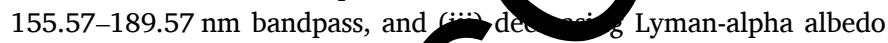
(199-125 nm). The ratio of the first ba dpasses, known as the "offband/on-band" ratio (referring the cont ssting UV spectral features of water frost in those regions), sho $n$ to be consistent with the presence of water ice layers $>0$ nm thick at locations where maximum surface temperaturec never a $110 \mathrm{~K}$. One such location is a patchy distribution of an the permanently shaded portion of Amundsen crater, wh shom combination of off/on-band ratios $>1.2$ and Lyman- $\alpha$ albedo 0.03 , indicating a possible water mass content of 0.1-2.0 (Hayne e al. 2015).

Some of these pata contained within our PSR target, but do not completely coincide with areas that also show high $1064 \mathrm{~nm}$ albedo (compare Hayne et al., 2015, Fig. 14 and Fisher et al., 2017, Fig. 11). Thus, while there is not complete overlap between these signatures, portions of our selected PSR target indicate annual maximum temperatures, as well as ultraviolet and near-IR signatures that are anomalous compared to other south polar terrain in permanent shadow, and are consistent with the presence of surface water frost. Our method intends to quantify any effect by the presence of water frost, or by ultra-low temperatures, on emis vity of these terrains in the far-IR, near their Planck peak $\left(\lambda_{\max }\right)$.

\subsection{Observations}

LRO's orbit configuration allows pportunit s for nadir observation of the two targets approximately eve two we ks, alternating between the day and night side. We retrim ations of both targets for the duration of the mission suly 20 -August 2017) and constrain them to emission angles $<5^{\circ}$, minimiz off-nadir effects.

We process observations n Divi er channels 7, 8 and 9, which are sensitive to photon vo veenrupproximately 25-41, 50-100 and $100-400 \mu \mathrm{m}$ respectiv $\mathrm{y}$, an $\mathrm{m}$ representative of surface brightness temperatures between 9 and 178, 43-69, and <43 K respectively (Paige et al., 2010 Thest provide sufficient spectral coverage of radiance due to ex ected nighttime temperatures. Data from channel 6 was foun b hot useful due to the channels very low sensitivity to PSR-relevant pperatures (predominantly sensitive to $\gtrsim 178 \mathrm{~K}$ ), and was therefore not included in this study.

For targets we plot brightness temperatures for Diviner channe 7,8 , an 9 as a function of local mean solar time (LMST) (Fig. 2). The spre $\mathrm{d}$ of brightness temperatures is expected to be due to the figh dys aty of diurnal and seasonal illumination conditions exp cienc d by polar terrain, that are heavily dependent on local topogbu cause shadows. This is particularly evident in the discontinuous distru is in daytime temperatures for the partially illuminated target (Fig. 2). Causes of the spread in apparent nighttime brightness temp. tures for both targets could include secondary emission from illuminared or warmer terrain, as well as be due to differences in surface temperature and emissivity. For the non-permanently shadowed target, esidual energy imparted by direct daytime illumination is also a likely ontributor.

\section{Method}

We aim to determine the distribution and extent of terrain that contributes to signal received by Diviner's long wavelength channels as fully and precisely as possible. To quantify the emitting surface area that contributes to the radiance recorded in each observation, we begin with the boresight of each observation, defined by the latitude and longitude of intersection with a lunar sphere of radius $1,737,400 \mathrm{~m}$ (Seidelmann et al., 2002).

Following Williams et al. (2016), we model the effective field-ofview (EFOV) as a probability distribution of signal that accounts for the instantaneous field-of-view, detector lag, and spacecraft motion during integration time. Details on how the Monte Carlo points that comprise each EFOV are transformed from instrument-relative to lunar body fixed coordinates, such that they can be projected onto a lunar digital elevation model (DEM), are described in Appendix A.

With the EFOV modelled as discrete rays connecting the lunar surface to the detector, we perform ray-tracing according to Teanby (2006) to determine the point of origin in the DEM from which each EFOV point originates. As an extension to the method for processing global gridded data records from modelled EFOVs described in Williams et al. (2016), our technique additionally allows processing of high emission angle observation geometries.

Due to the nature of the processing pipeline a case exists where observations or partial observations may be excluded from analysis when the boresight of a very high emission angle observation $\left(\gtrsim 80^{\circ}\right)$ does not intersect with the lunar sphere, but a fractional part of the EFOV of the observation does, e.g. the boresight passes the lunar limb, but e.g. a 

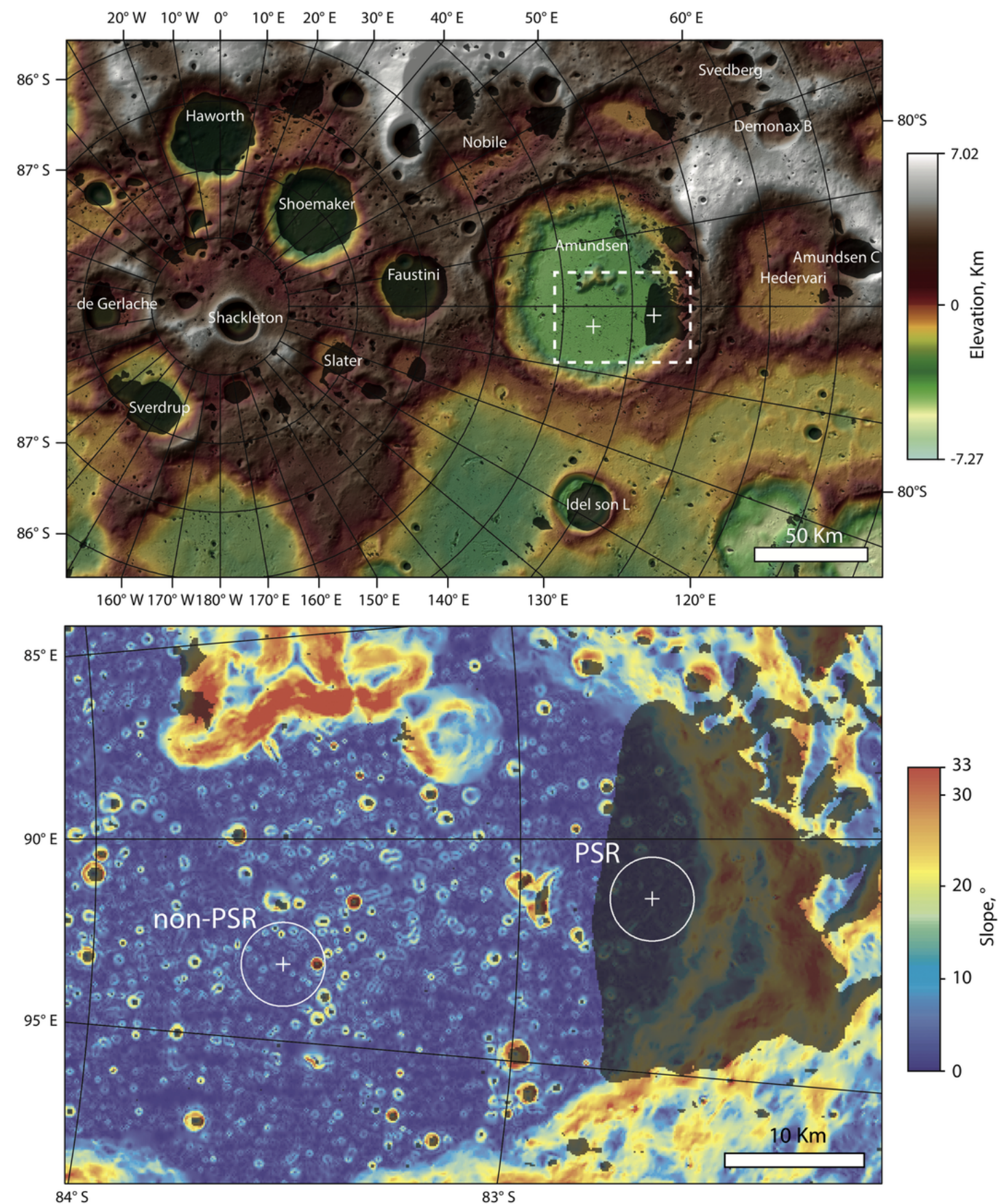

Fig. 1. Top - Lunar south polar region in 9 A

A $3 \mathrm{~km}$ radius from the targets is marked, used to ure legend, the reader is referred to th

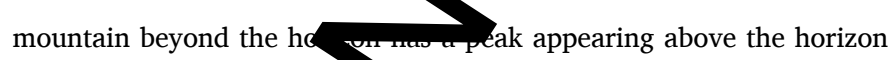
that contributes some signa on these cases the whole observation is excluded. However, cove ove targets by the available data at high emission angles is poor ( Section 5.3).

The terrain modo is prod ced as follows: A triangular mesh is first generated on a lunar spmure of radius $1737.4 \mathrm{~km}$ (Seidelmann et al., 2002) using the icosahedron subdivision generation method of Teanby (2006). Vertices result from 12 iterative bisections of icosahedron edges, corresponding to a mean on-sphere vertex separation of $\sim 560 \mathrm{~m}$ and triangle area of $\sim 0.14 \mathrm{~km}^{2}$ (Sefton-Nash et al., 2017). Slopes be- low this scale are not resolved, but the mesh resolution is on the order of the size of Diviner observation footprint. After generation of mesh vertices on the lunar sphere, vertices are adjusted in the vertical direction by retrieving the elevation at each vertex's on-sphere position via interpolation of the LOLA gridded data record LDEM_60S_60M.IMG (Smith, 2017) using Delaunay Triangulation (Delaunay, 1934).

At high emission angles, ground-projected EFOVs are extended in the horizontal direction of the look vector, including distal terrain that may be of significantly different radiative state. This sometimes results in the location of maximum probability, i.e. where the peak magnitude 
PSR target
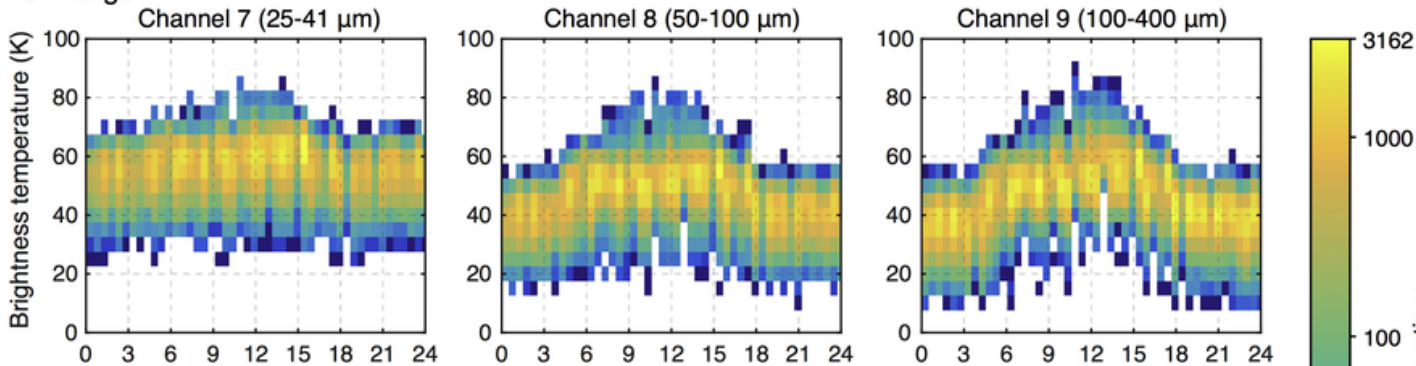

non-PSR target
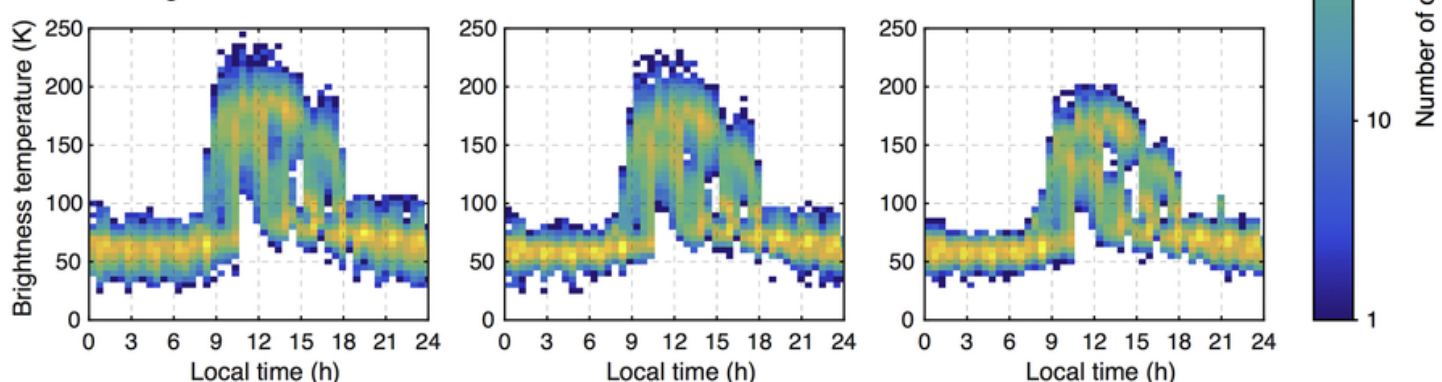

Fig. 2. 2D histograms of brightness temperatures observed for the permanently shadowed (top) and tially illum 2009-August 2017), and plot as a function of local mean solar time (LMST). To best illustrate structure in distrib $\log$ color scale) in bins of $5 \mathrm{~K}$ and $0.5 \mathrm{~h}$. To reduce off-nadir effects data with boresight emission end, the reader is referred to the web version of this article.)

of the signal originates, being significantly offset from the boresight intersection with the surface. To minimize potential scatter, we therefore discount observations where the location of peak EFOV probability ir tersects with terrain outside the target radius.

Conversely, we also account for observations whose boresigb rain intersections may lie outside the target radius, but whose haximum EFOV probability lies inside. This is accomplished by initiall 1 culating EFOVs for data whose boresight intersection is then including any with an EFOV maximum that lies with

For each of the Diviner thermal channels 7, 8 and their sensitivity to PSR and nighttime temperatures) age of radiance was calculated for each triangle in where observations in each orbit track intersect within one orbit are spaced closely enough in tively they represent the same surface radiativ observations from the three relevant therm cha basis such that they may be associated. To illus the cumulative result of this process, we plot the total nu rations made by channel 9 of the two targets on a per ty angle bas (Fig. 3). In general, more observations were acquired of th non-PSR arget $(\sim 8500-9200$ per triangle) than for the PSR target ( mild heterogeneity in spatial co crage of the two target areas is evident. We propagate the number fobserva ons as a function of various parameters in our analysis in ord to hig ight any potential observation bias.

Radiance, $\mathfrak{R}$, received in a chanı $c$, is described by:

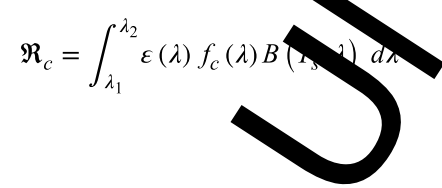

where $f_{c}(\lambda)$ and $\varepsilon(\lambda)$ are the quantum efficiency and emissivity at wavelength $\lambda$, respectively, $B$ is the black body radiance emitted at wavelength $\lambda$, by a surface of temperature, $T_{s}$. Wavelength limits on integration, $\lambda_{1}$ and $\lambda_{2}$ encompass the range for which quantum efficiency data

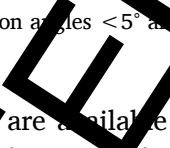

ted (bottom) targets over the duration of the LRO mission (July ons we have selected to display the number of observations (on a ed. (For interpretation of the references to color in this figure legare ala e (Paige et al., 2010a) for each channel. This formulation ignores cuission angle effects.

For a given observation geometry, we may make two important assertins: Firstly, the quantum efficiency as a function of wavelength, $f_{c}(\lambda)$, is constant and known for each channel (Paige et al., 2010b). Secndly, $B\left(T_{s}, \lambda\right)$ may be assumed constant for a fixed location that is oberved by Diviner in a single orbit pass. Diviner's integration time is $0.128 \mathrm{~ms}$ and the resulting time differential between successive observations between channels with overlapping EFOVs is $<1 \mathrm{~s}$. Changes in the radiative state of the surface are therefore unlikely over the course of a few integration times, and consequently the surface kinetic temperature, $T_{s}$, is assumed fixed during an orbit pass.

Typically for each orbit, co-aligned observations from all three long wavelength thermal channels (7, 8 and 9) are coincident on the DEM. In these cases, where radiance is recorded in multiple spectral bandpasses, we may approximate $T_{s}$ with the bolometric brightness temperature, $T_{B O L}$ (Paige et al., 2010a). $T_{B O L}$ is retrieved from a black body spectrum that is fit to observed radiance integrated over multiple bandpasses. Importantly, this is performed for every terrain element that contributes signal in the orbit swath. $T_{B O L}$ is, by definition, a brightness temperature, in that it assumes black body emission i.e. it includes the cumulative effects of any dependence of emissivity on temperature or wavelength.

The orientation and position of each terrain element produces an individual observation geometry, an important parameter of which is the terrain relative emission angle. Therefore, for each terrain element, i.e. triangle in the DEM, that is observed, and over many orbits, we generate a large list of data points to populate a parameter space with dimensions: $T_{B O L}, \mathfrak{R}_{7}, \mathfrak{R}_{8}, \mathfrak{R}_{9}$, local time and terrain relative emission angle.

\section{Results}

Observed radiance includes any effects of variation in emissivity as a function of local time, emission angle or bolometric temperature. Consequently, without independent constraint of $\varepsilon(\lambda)$ in any channel 


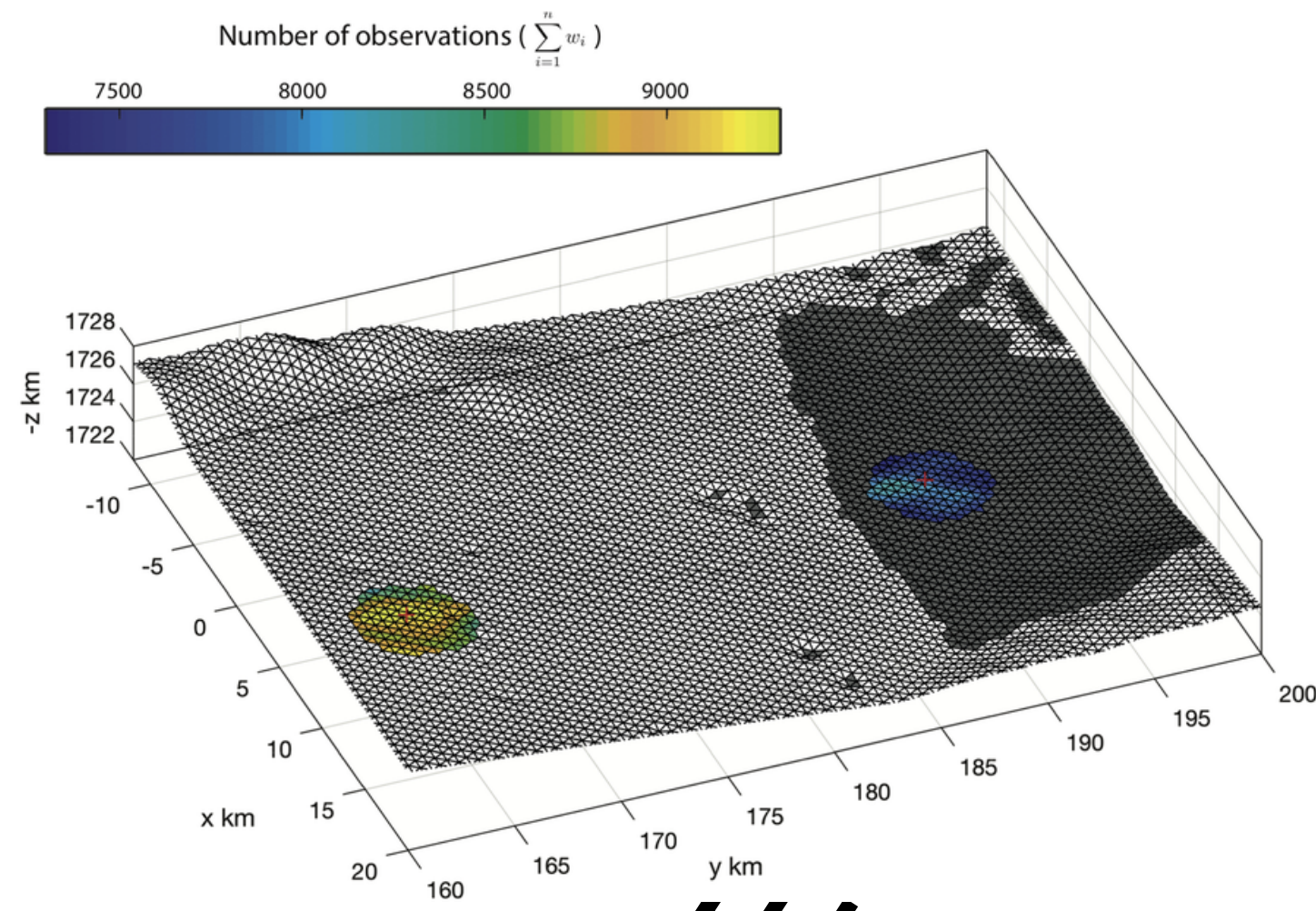

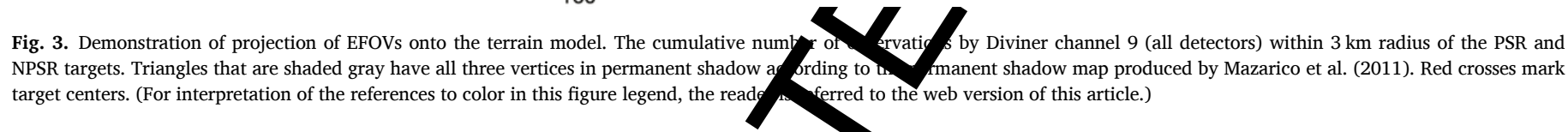

we cannot deconvolve the effects of surface kinetic temperature apparent emissivity using only radiance received in a single chann

With two bandpasses, however, we may calculate the change in apparent emissivity. Using this rationale, we inves ${ }^{\prime \prime}$ gate ratio of radiance received in Diviner channels 8 and $9, \mathfrak{R} / 9$, for the permanently shadowed and partially illuminated target. D to fac that $T_{B O L}$ is calculated using radiance from channels 7, 8 and grap ing the ratio $\Re_{8 / 9}$ against $T_{B O L}$ reduces self-dependeng es at cots be introduced by instrumental effects if only channels and thoused to calculate $T_{B O L}$.

However, while interesting trends may be ob ind in apparent correlation between $\mathfrak{R}_{8 / 9}$ and bolometric temp ratur ing. 4), they are not meaningful in terms of extracting inform. on apparent emissivity unless we understand what value of $\mathfrak{R}_{\text {Q10 }}$ sho $\mathbf{H}_{\mathrm{d}}$ be expected as a function of surface kinetic temperature ve mu accunt for the convolution of Diviner's spectral bandpas s with ra iance from thermal emission.

\subsection{Band ratio model}

To address this we model 9 dian exp ted as a function of surface kinetic temperature using $T_{B O L}$ calculate expected radiance from a black body by multiplyin antum efficiency for each channel by the black body curv $f(\lambda) B\left(T_{s}, \lambda\right)$ (Fig. 5, left, middle). Over a PSR-relevant range of $(0-20, \mathrm{~K})$, and for each Diviner channel, we integrate beneath these curn to model radiances expected for a black body ( $\varepsilon$ is not wawnth dep hdant and always equal to unity):

$\Re_{c, \text { model }}=\varepsilon \int f_{c}(\lambda) B\left(T_{S}, \lambda\right) d \lambda$

Ratios between these integrated radiances are plotted as a function of temperature (Fig. 5, right). We select channels 8 and 9 for this inves- tigation, as they are most sensitive to PSR-relevant temperatures and allow us to best explore the ultra-low temperature regime of the PSR arget.

For the observed band ratio, $\mathfrak{R}_{8 / 9}$, we permit wavelength-dependent emissivity, $\varepsilon(v)$. The modelled band ratio $\mathfrak{R}_{8 / 9 \text { model }}$, can therefore be compared to the observed $\mathfrak{R}_{8 / 9}$ :

$$
\begin{aligned}
\mathfrak{R}_{8 / 9}-\mathfrak{R}_{8 / 9 \text { model }}= & \frac{\int \varepsilon(\lambda) f_{8}(\lambda) B\left(T_{S}, \lambda\right) d \lambda}{\int \varepsilon(\lambda) f_{9}(\lambda) B\left(T_{S}, \lambda\right) d \lambda} \\
& -\frac{\int f_{8}(\lambda) B\left(T_{B O L}, \lambda\right) d \lambda}{\int f_{9}(\lambda) B\left(T_{B O L}, \lambda\right) d \lambda}
\end{aligned}
$$

Assuming that bolometric brightness temperature is a valid representation of surface kinetic temperature, i.e. $T_{B O L}=T_{S}$, then the difference between the two is influenced only by any differences in $\varepsilon(\lambda)$ that might exist between the apparent emissivity integrated over the bandpasses of each channel.

In our formulation, with $\mathfrak{R}_{8}$ as the numerator, a positive value of $\mathfrak{R}_{8 / 9}$ implies that emission received by channel 8 increases relative to that in 9 , or conversely that emission received in channel 9 decreases relative to that in 8 . Consequently, because $\mathfrak{R}_{8 / 9}$ model represents a black body where emissivity is therefore constant at all wavelengths, a positive value of $\mathfrak{R}_{8 / 9}-\mathfrak{R}_{8 / 9 \text { model }}$ corresponds to an under-prediction by the model of the apparent relative increase in emissivity over the channel 8 bandpass compared to that in 9 , or vice versa, i.e.:

$\int \varepsilon(\lambda) f_{8}(\lambda) d \lambda>\int \varepsilon(\lambda) f_{9}(\lambda) d \lambda$ 

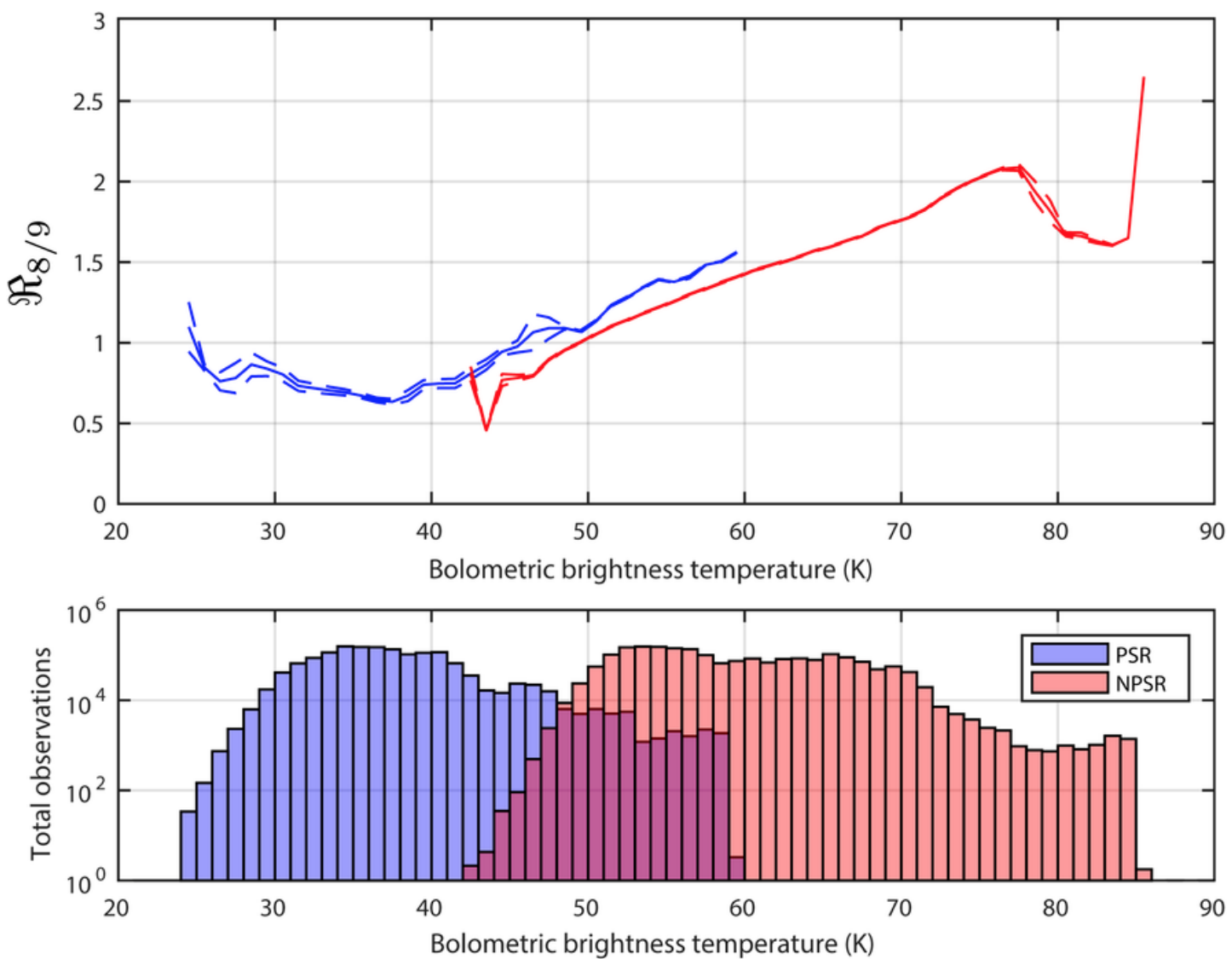

Fig. 4. Top - Weighted mean $\Re_{8 / 9}$ as a function of bolometric brightness temperat the distribution of $\Re_{8 / 9}$ at each value of $T_{B O L}$. Bottom - Total number of observati tation of the references to color in this figure legend, the reader is referred to the

. Dashed lines for the PSR (blue) and NPSR (red) curves represent the standard deviation on (sum of servations in channels 8 and 9), plotted as a function of $T_{B O L}$ in bins of $1 \mathrm{~K}$. (For interpreversion o his article.)
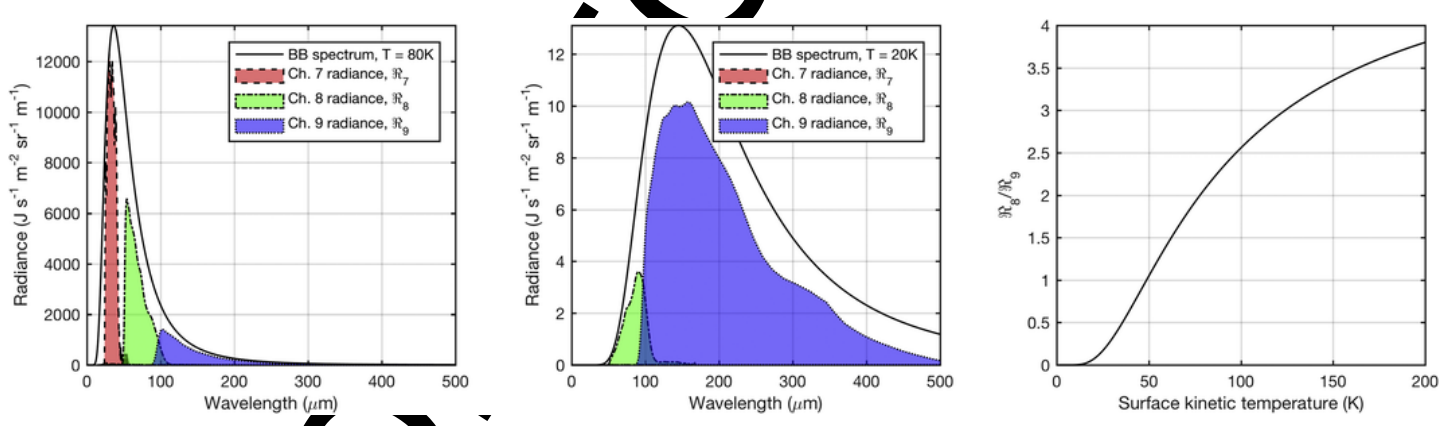

Fig. 5. Left: Spectrum for a black body at $80 \mathrm{~K}$ th bandpass nels 8 and 9 in this case, $\mathfrak{R}_{8 / 9}=2.06$. Center: Sir tarly, spect temperature, emitted radiance is about a facto

face kinetic temperature. Channel 9 radia

of Diviner channels 7 (red), 8 (green) and 9 (blue) integrated beneath it. The ratio of signal contribution between chan$\mathrm{m}$ emitted by a black body at $20 \mathrm{~K}$ and the corresponding integrated contributions from channels 7, 8 and 9 . At this low at $80 \mathrm{~K}$, but the signal is dominated by channel 9 , with $\Re_{8 / 9}=0.07$. Right: $\mathfrak{R}_{8 / 9}$ plotted as a function of black body sur-

the references to color in this figure lege the reade s referred to the for surface kinetic temper.

With this rationale estabhed, the mean $\mathfrak{R}_{8 / 9}-\mathfrak{R}_{8 / 9}$ model against bolometric brightness tem rature that we observe for the PSR and NPSR targets (Fig. ture domains for the two rgets, due to their different nighttime temperature regimes (Fig. 2). Th largest number of observations for the PSR target is betwe largest number of observations for the the NPSR target between $-70 \mathrm{~K}$ (Fig. 6). We deem these tempera-
ture intervals therefore to be the least susceptible to any remaining observation bias in other parameters that correlate with temperature. We explore possibility of observation bias due to emission angle or local time coverage in the Discussion section.
For the NPSR target, importantly we see that in the temperature domain with the strongest statistics, $\sim 50-70 \mathrm{~K}$, observations are almost perfectly predicted by our model, i.e. $\mathfrak{R}_{8 / 9}=\mathfrak{R}_{8 / 9}$ model . This implies that relative emissivities integrated over the two channels are equal, though not necessarily 1 , as in the black body model. It also implies that relative emissivities between the channels stay constant over this temperature range.

A small upturn around $75 \mathrm{~K}$ and subsequent dramatic downturn from $\sim 77 \mathrm{~K}$ upwards both coincide with reduced numbers of observations, and therefore more susceptible to observation bias. Consequently, we do not interpret these features as having significant physical meaning. 

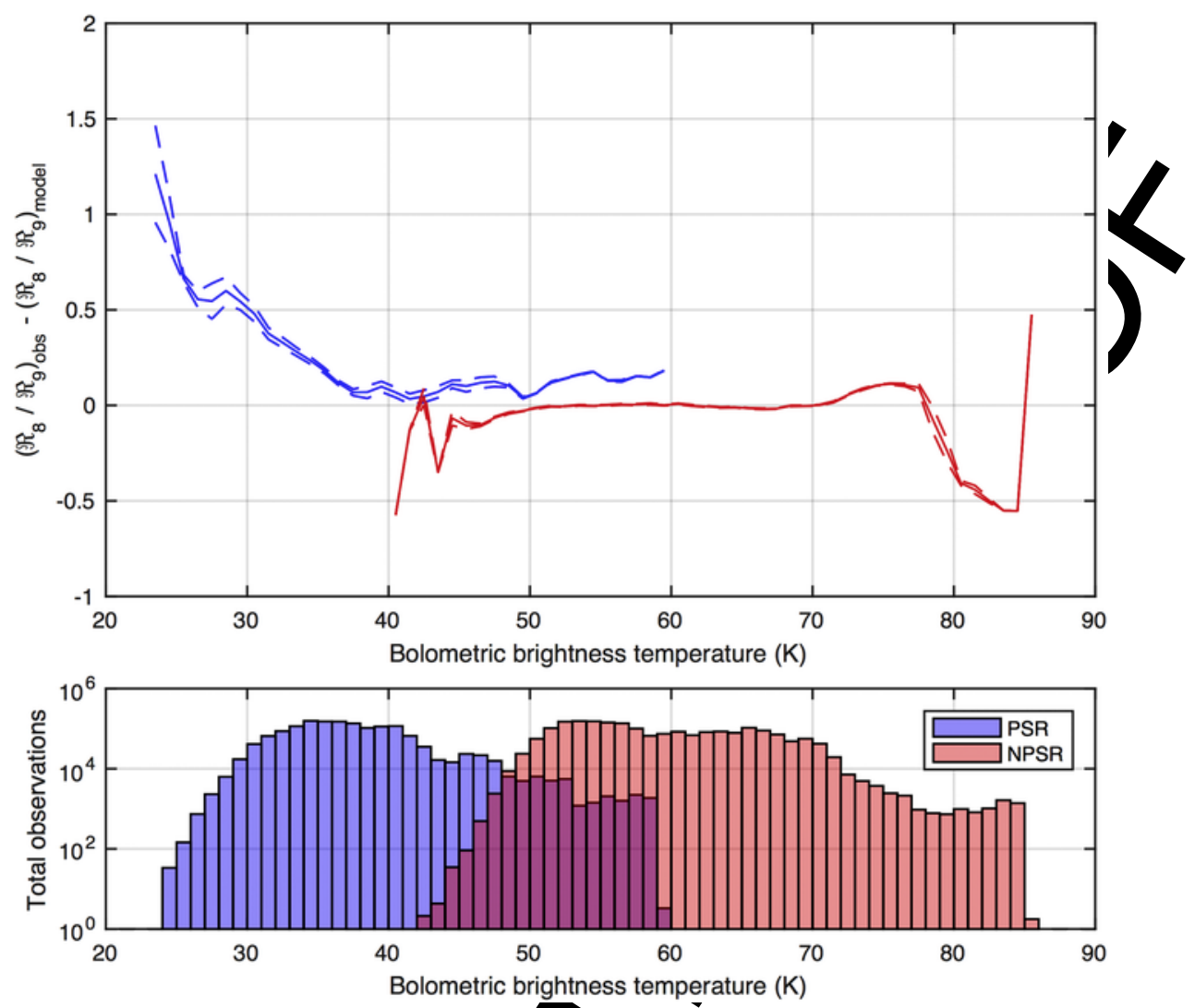

Fig. 6. Top $-\mathfrak{R}_{8 / 9}-\mathfrak{R}_{8 / 9 \text { model }}$ as a function of bolometric brightness temperature for edh targe ashed lines indicate a 1?? envelope. Bottom - Total number of observations in both channels 8 and 9 (log scale) for each target, plotted as a function of $T_{B O L}$ in bins of 1

For the PSR target we see different emissive behavior. For th temperature region between $40 \mathrm{~K}$ to $\sim 60 \mathrm{~K}, \mathfrak{R}_{8 / 9}-\mathfrak{R}_{8 / 9 \text { model }}$ is and higher than that for the NPSR target. Implying that diff sivities are measured in the two bandpasses. However, this range suffers from reduced numbers of observations com NPSR target, decreasing to just a few hundred observg wards $60 \mathrm{~K}$, so this result should be interpreted with

Most importantly for the target in permanen $\mathrm{bad}_{\mathrm{d}} \mathrm{w}$, between 30 and $40 \mathrm{~K}$ where observation density is highest a fem $10^{5}$ observations per bin; equivalent to the highest data de sitie far the-NPSR target), a slope is seen in relative emissivity b ween chansels 8 and 9: $\mathfrak{R}_{8 / 9}-\mathfrak{R}_{8 / 9 \text { model }}$ appears to increase with dec ing temperature in this range.

This major result implies a temper ure-depe dent apparent emissivity in far-IR for the PSR targe Either, emissivity between 50 and $100 \mu \mathrm{m}$ increases relative to $100-10 \mathrm{~m}$ or emissivity over the longer wavelength bandpass decr ases relative to that in the shorter.

\section{Discussion}

\subsection{Far-IR spectral features of waten}

To explore possible terph our observations, we first consider the far IR spectral tures of water ice, and the likelihood of their role in the ove result Amorphous water-ice has a broad absorption peak near am, aile crystalline ice has absorptions near $44 \mu \mathrm{m}$ and $62 \mu \mathrm{m}$ (Moore and Hudson, 1992). The sensitivity and spectral coverage of Diviner channels 7 and 8 might permit detection of these features, though complexities are introduced by the presence of silicates. Absorption is sensitive to grain size, crystallinity, contaminants and the porosity of water ice (Hayne et al., 2014, Carvano et al.,
006). The bands at 44-45 are most likely to yield a detection for specral mixtures of ice and regolith, however this region is poorly detectable by Diviner because it is located near the upper sensitivity boundary of channel $7(\sim 41 \mu \mathrm{m})$, but lower sensitivity boundary of channel $8(\sim 50 \mu \mathrm{m})$ (Paige et al., 2010a, 2010b). In PSRs where surface water frost is most likely to be present, temperatures are coldest and thus measurements more challenging. Consequently, more spectral channels with narrower bandpasses and higher sensitivity would probably be needed to distinguish these features from thermal emission from ice-poor lunar regolith.

\subsection{Sub-FOV thermal heterogeneity due to roughness}

Accepting that composition-based spectral features of water ice are not likely to significantly contribute to the signal we observe, we next consider the possible effects of surface roughness in PSRs, as has been quantified using in TIR observations over the lunar globe (Bandfield et al., 2015). Sub-FOV scale "micro" doubly-shadowed cold traps, topographic lows in PSRs that are also protected from secondary and higher order thermal emission (e.g. from crater walls), may contribute to anisothermality in PSRs. We construct a simple model that considers radiance contributions from a bi-modal brightness temperature distribution. The black body emission at wavelength $\lambda$ from two bodies at temperatures, $T_{1}$ and $T_{2}$, may be described by:

$$
\mathfrak{R}_{\text {total }}(\lambda)=a B\left(T_{1}, \lambda\right)+(1-a) B\left(T_{2}, \lambda\right)
$$

where $\mathfrak{R}_{\text {total }}(\lambda)$ is the total radiance and $a$ is the fraction of the radiance originating from the body at temperature $T_{1}$. Multiplying $\mathfrak{R}_{\text {total }}(\lambda)$ with the quantum efficiencies of each channel $\left(f_{8}\right.$ and $\left.f_{9}\right)$ and integrating over wavelength, we retrieve the expected band ratio for the ther- 
mal emission spectrum modelled for a bimodal temperature distribution:

$\mathfrak{R}_{8 / 9}^{\prime}=\frac{\int f_{8}(\lambda) \mathfrak{R}_{\text {total }}(\lambda) d \lambda}{\int f_{9}(\lambda) \mathfrak{R}_{\text {total }}(\lambda) d \lambda}$

$T_{1}$ represents the temperature of the double permanently shadowed terrain, and is varied between 15 and $40 \mathrm{~K} . T_{2}$ represents the temperature of the single permanently shadowed terrain, which we fix at $40 \mathrm{~K}$, typical for the PSR target (Fig. 2).

Subtracting the band ratio modelled for the bimodal temperature distribution, $\mathfrak{R}_{8 / 9}^{\prime}$, from that for a uniform temperature distribution at $40 \mathrm{~K}$ (Fig. 7) permits us to assess the potential effects that sub-FOV doubly-shadowed terrain could have on band ratios.

We plot the difference between $\mathfrak{R}_{8 / 9}$ expected for observations of terrain at $40 \mathrm{~K}$ and that for the same terrain with some fraction at a lower temperature $\mathfrak{R}_{8 / 9}^{\prime}$, due to its double-shadowing. We vary both the fraction of colder terrain (from 0.4 to 1 ) and its temperature from 15 to $40 \mathrm{~K}$. Fig. 8 shows the difference is larger when anisothermality and fraction of doubly shadowed terrain is greatest, but can also reduce for moderate fractions $(\sim 0.5-0.8)$ of very cold $(\sim 15-25 \mathrm{~K})$ doubly shadowed terrain. This is due to the non-linearity of Planck emission to the signal with decreasing temperature. We acknowledge the model described considers a bimodal temperature distribution, and that a realistic temperature distribution would be required to fully assess the possible magnitude of this effect for real observations. Nonetheless, it is clear that terrain with brightness temperatures of $\sim 40 \mathrm{~K}$, such as the PSR target (Fig. 2), could produce anisothermality observable in the far-IR if doubly-shadowed terrain occupies mode significant fractions of FOVs. Indeed, Fig. 6 indicates valy $\mathcal{S}$ of $\mathfrak{R}_{8 / 9}-\mathfrak{R}_{8 / 9 \text { model }}$ of $0-0.5$ between 30 and $40 \mathrm{~K}$, where statisti are best. Such values could be produced if PSR terrain at $40 \mathrm{~K}$ conto. more than about 0.4 areal fraction of terrain that is up to (Fig. 7).

Modelling by Rubanenko and Aharonson (2017) [F that terrains with high RMS slope $\left(\sigma_{S}\right)$ distribution pro uce honer cold-trap areal fractions, and by the same principle buld $S_{\mathrm{SO}}$ duce higher areal fractions of doubly-shadowed PSRs. For s netically generated terrains with Gaussian slope distributior approximate latitude of the two targets in this stud permits PSR areal fractions of up to $\sim 0.45$, and relatively smooth surfaces $\left(\sigma_{S}=10^{\circ}\right)$ are required for PSR areal fractions of up to $\sim 0.3$.

Therefore the apparent temperature dependence shown in Fig. 6 could be caused by binning of observations a function of temperature, such that lower apparent temperaty as apserved for areas with higher areal fractions of double-PSRs th fOV. For the apparent temperature range $30-40 \mathrm{~K}$, this suggests our servations of the PSR could be consistent with it containing codes ro rogh surfaces comparable in RMS slope distribution to fose deriv d for global lunar TIR observations (Bandfield et al., 2015)

However, it is unlikely that suld be sufficient to produce the high values of $\mathfrak{R}_{8}-\mathfrak{R}_{8 / 9}$ del observed for temperatures $\precsim 35 \mathrm{~K}$. For example, doubly hadowed errain at $20 \mathrm{~K}$ would need to occupy $84 \%$ of the FOV (when the sur unding terrain is $40 \mathrm{~K}$ ) (Fig. 7) to produce $\mathfrak{R}_{8 / 9}-\mathfrak{R}_{8 / 9}$ ded 0.2 ovserved in Fig. 7 .

For apparent tem ratur of $\mathrm{K}$ the fewer number of observations of the PSR targen ts in poorer statistics, as evidenced by the widening of the stard a ration envelope (blue dashed line in Fig. 6). It is possible nat $t$ reduction in SNR with temperature for channel 8 makes vas $\mathfrak{R}_{8 / 9}$ more spurious for apparent temperatures $\precsim 20 \mathrm{~K}$. Nonetheless, ven if we had confidence in values of $\mathfrak{R}_{8 / 9}$ at such temperatures, in the case of our model it is very unlikely that surface roug citeon ould be the cause of $\mathfrak{R}_{8 / 9}-\mathfrak{R}_{\frac{8}{9} \text { model }} \gtrsim 0.5$.

\subsubsection{In ication of micro-cold traps}

While the stence of doubly-shadowed micro cold traps may come no arprive given our understanding of lunar surface roughness bu ield al., 2015), these results could indicate that observations of PSh Diviner contain thermal emission from ultra-cool surfaces hat could be $10-15 \mathrm{~K}$ cooler than their surroundings.

cold-trapped volatiles with an impact origin, selected compounds with low volatility temperatures from the distribution of those known to be present in comets (e.g. Cottin et al., 1999; Ehrenfreund nd Charnley, 2000; Task Group on Organic Environments in the Solar System, 2007) and meteorites (Botta and Bada, 2002), such as simple organics and clathrates (Zhang and Paige, 2009), may be more relevant to the possible volatile inventory in these cooler micro cold traps.

As an example for one species, Hodges (1980) explored the processes by which exospheric argon could be cold-trapped in doublyshadowed areas. Argon's cold-trapping temperature was found to be between $\sim 40$ and $\sim 70 \mathrm{~K}$ depending on the substrate onto which it is adsorbed. Zhang and Paige (2009) found the temperature criterion for long-term stability of argon ( $<1 \mathrm{~m}$ of sublimation per billion years) to

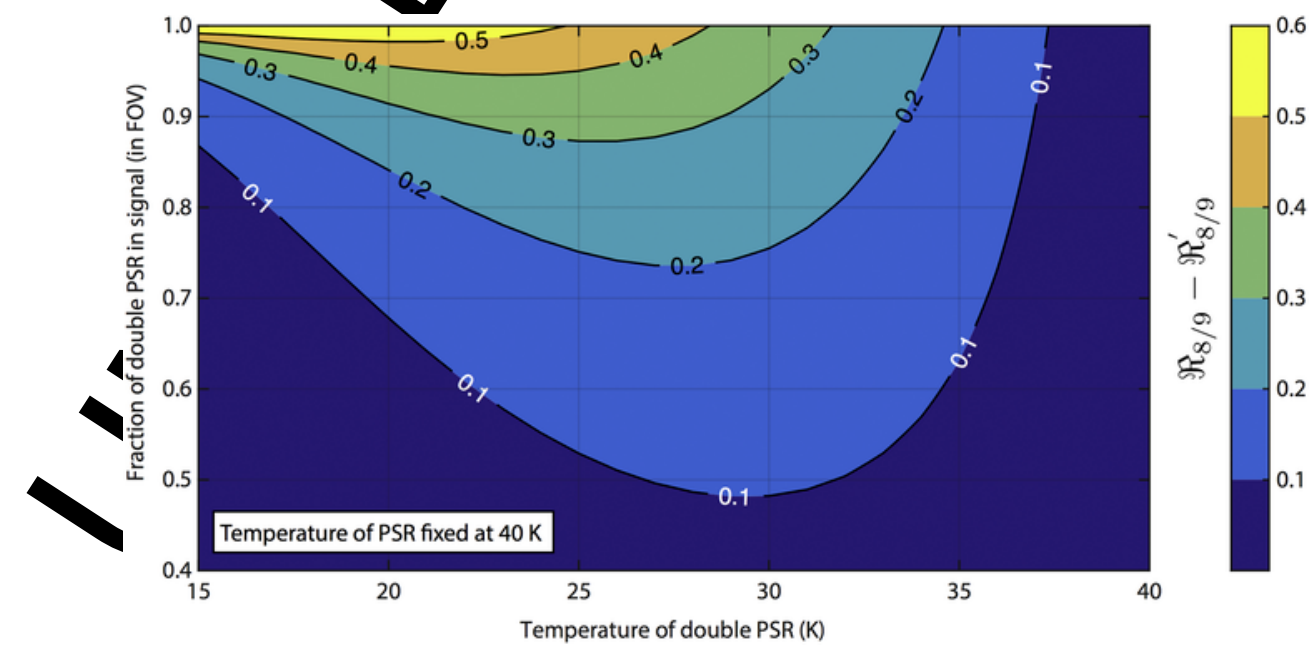

Fig. 7. Difference between $\mathfrak{R}_{8 / 9}$ modelled for a black body with uniform temperature at $40 \mathrm{~K}$ and that modelled for a surface with some fraction at a lower temperature, $\mathfrak{R}_{8 / 9}^{\prime}$, plotted as a function of both the fraction of ultra-cold terrain in the field of view, and its temperature. 


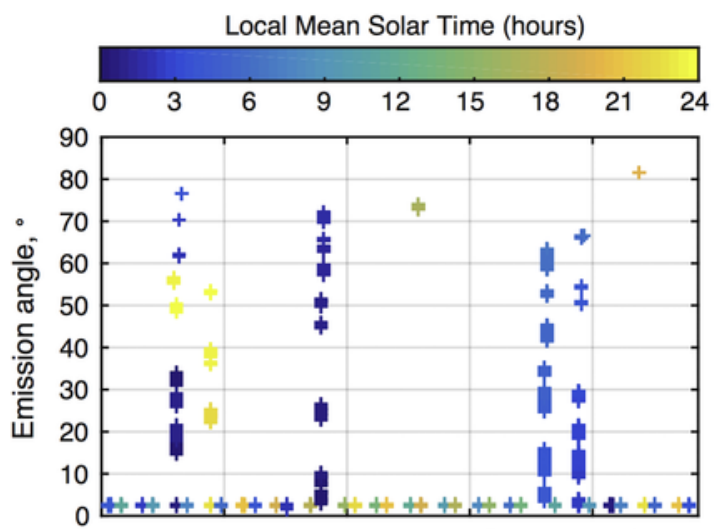

2016-Jan 2016-Apr 2016-Jul 2016-Oct 2017-Feb
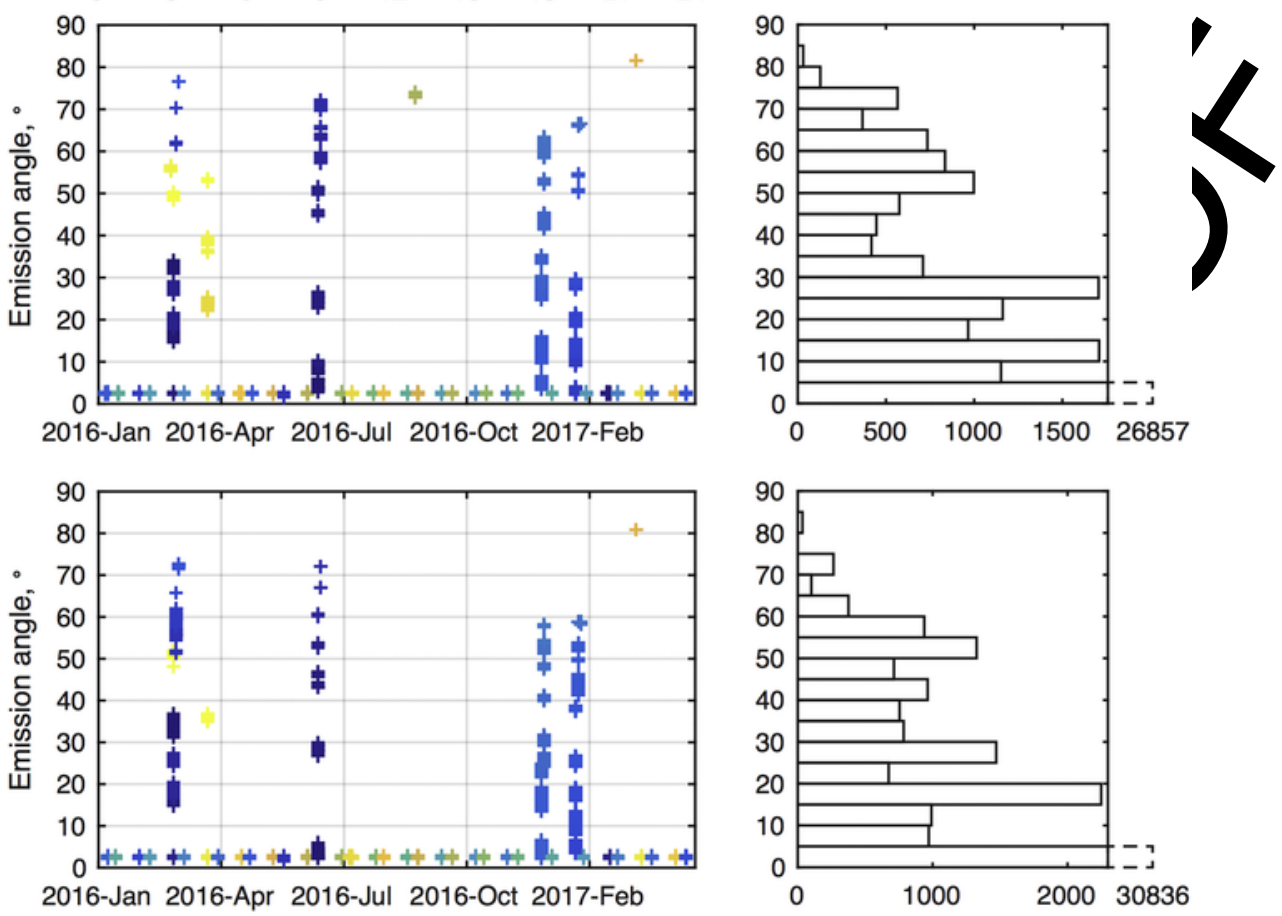

Date

Number of observations in Ch. 9

Fig. 8. Left - Date and emission angle of the boresight vectors for observations of the period is selected to highlight high emission angle observation campaigns. Channel 9 is most identical coverage. Note that high emission angle targeted observations were scl- led at night only. Right - Distribution of emission angle coverage for the two targets. Emission

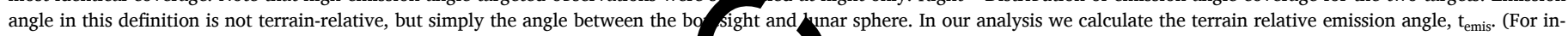
terpretation of the references to color in this figure legend, the reader is referred

the web ven of this article.)

be $\sim 20 \mathrm{~K}$. In the same study they asserted that, for organic co pounown from a forward-looking part of a channel 8 EFOV, i.e. that is inclined volatility increases roughly linearly with molecular mas that such complexities should be expected for all species the emplacement and loss processes for volatiles in sipr shadowed regions.

We assert that micro-cold traps may contribute the sserved differences between modelled and observed $\mathfrak{R}_{8 / 9}$, but the vre not likely to be the sole cause. Thus we proceed to exp ore lternan potential sources of observation bias and effects that could caur changes in emission that may collectively combine to reps it the observed 'apparent' emissivity.

\subsection{Emission angle}

The vast majority of observat tive emission angles $\left(t_{\text {emis }}\right)$ for bd $h$ targets. acquired at $t_{\text {emis }} \leq 3^{\circ}$ and $\sim 91 \%$ ere acqy some observations at higher ission wes were acquired as part of a specific campaign involved 'lock-o targeting, where Diviner actuators were moved in intervals respacenaft passed over the targets to observe at a range of emicion s. These observations began as part of a campaign in March 20, ana visible in Fig. 8 as observations closely spaced on the time ax

To illustrate tre ds over emission angle, we plot $\mathfrak{R}_{8 / 9}-\mathfrak{R}_{8 / 9 \text { model }}$ as a funturn of $\mathrm{t}_{\mathrm{emis}}$ (Fig. 9).

$t_{\text {emis }}$ is specific to each triangular facet in the terrain model, and may differ between channels 8 and 9 , even for observations in the same orbit. This is due to the spread of emission angles in EFOVs. To demonstrate; consider that some signal in an observation originates from the boresight towards the spacecraft velocity vector. The same terrain (i.e. the same triangle in the DEM) may also radiate and contribute signal to the backward-looking part of a channel 9 EFOV. If only these portions of observations were binned, then the band ratio would not account for the difference in emission angle. It is important to reiterate however, that the fringes of EFOVs contribute far less signal to observations than the central portions. Consequently, while there is some scatter in the band ratio caused by the spread of emission angle within EFOVs, the origin of overall signal is heavily weighted towards the boresight, where emission angles in EFOVs from different channels are most similar.

Nonetheless, to quantify scatter in band ratio that may be caused by this effect, we calculate the $1 \sigma$ on the distribution of emission angles in each bin (Fig. 9), and find in general that errors are comparable in size or smaller than bin width, equal to $2^{\circ}$ in our analysis. Increased scatter at high emission angles, indicated by widening of the $1 \sigma$ envelope in the distribution of $\mathfrak{R}_{8 / 9}-\mathfrak{R}_{8 / 9 \text { model }}$ may be due to the combined effects of fewer observations at high emission angles, and the enlargement of the EFOV at high emission angles. With increasing emission angle, signal is contributed from wider areas, increasing the probability of mixing of thermal states within each observation and thus, spread in the calculated band ratios.

Theory suggests that for small values of the single scattering albedo, $w$, directional emissivity, $\varepsilon_{d}$, is 1 at all angles and the surface emits like a black body (Hapke, 2012). Therefore low-albedo materials can be assumed to have emissivity that is not dependent on emission angle. This is supported by measurements of sand and playa surfaces by Jankowsky and Jacobson (1990). Our observation of invariant emissiv- 

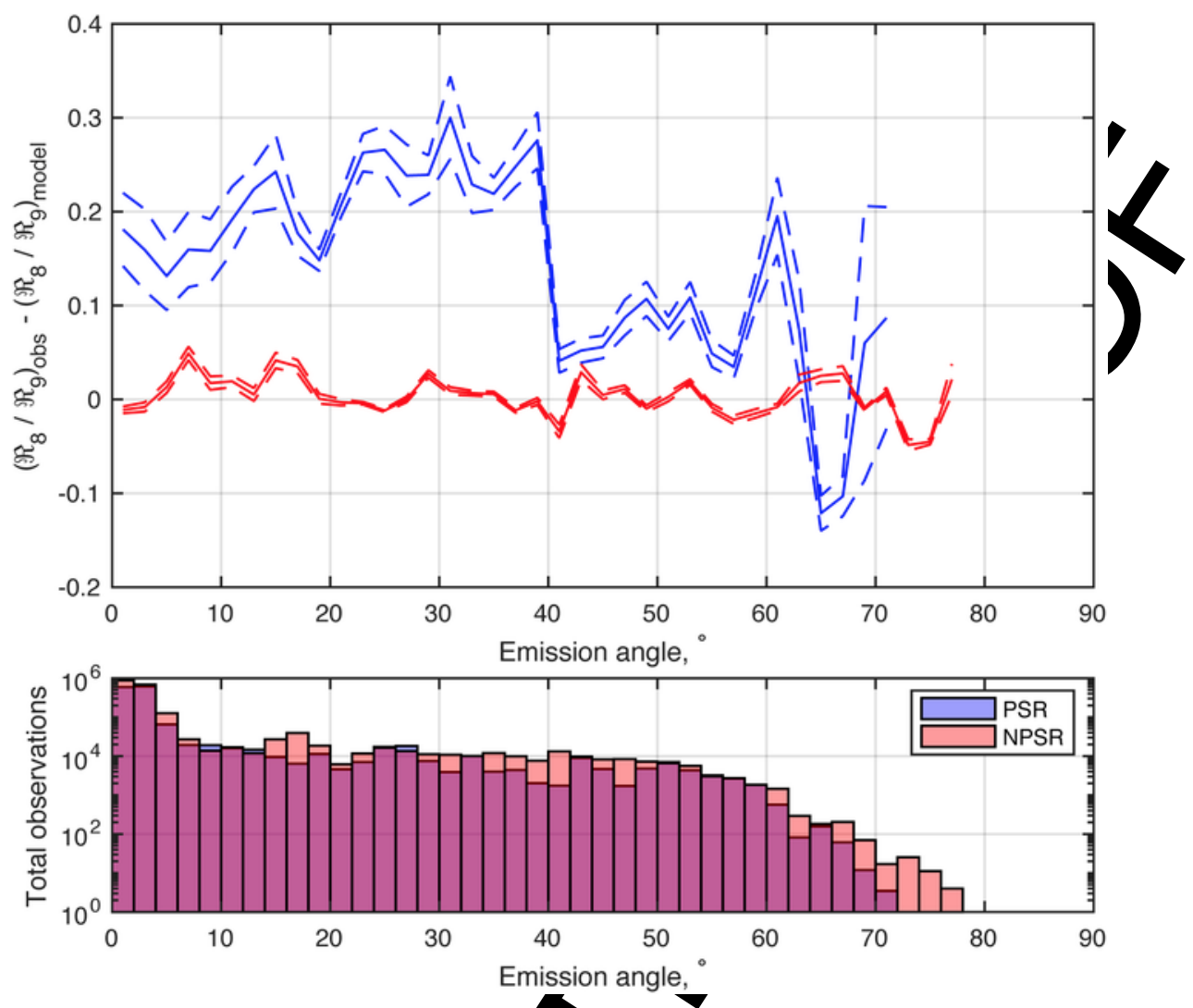

Fig. 9. Top $-\Re_{8 / 9}-\Re_{8 / 9 \text { model }}$ as a function of terrain-relative emission angle ( $\mathrm{t}_{\text {emis }}$ ) fd eachi both channels 8 and 9 , for each target, plotted as a function of $t_{\text {emis }}$ in bins of $2^{\circ}$.

ity over emission angle of the NPSR target is generally consisten with it being made of a relatively low albedo material, but poor cover at high emission angles (where a downturn in $\varepsilon_{d}$ would be m nent) precludes meaningful constraint of albedo.

While both targets suffer from greatly reduced coven hig emission angles $\left(\succsim 55^{\circ}\right)$, the PSR target exhibits a $\mathfrak{R}_{8 / 9}-\mathfrak{R}_{8 / 9 \text { model }}$ for $\mathrm{t}_{\mathrm{emis}} \succsim 40^{\circ}$. The region between cident with a reduction in observation coverage, br the overall number of lock-on observations in the oft- , ir campaign makes this relationship sensitive to other releva $\Omega$ p amete that may influence $\mathfrak{R}_{8 / 9}$, and precludes meaningful co paris $\widetilde{A}_{\text {a }}$ inst a global nighttime emissivity trends (Bandfield et al., 1 , which result from averages of the global dataset and thus so the bias that could be introduced by local time cov age of servations. Next, we investigate possible observation bias ca sed by loc 1 time.

\subsection{Local time}

The relationship between loca time an ing (Fig. 10). Observation bu in mo expected to contribute to any trend, due to the serally even coverage of all nighttime hours for both targe

$\mathfrak{R}_{8 / 9}-\mathfrak{R}_{8 / 9 \text { model }}$ fo the - -PSR target exhibits no apparent dependence on local time and ws anly small deviations from 0 over the entire lunar night consisten, with invariance in the far-IR emissivity for this target; also a arent a function of temperature and emission angle (Figs. 6 and 9).

Results for the PSR target are dramatically different, however. Short timescale noise on the difference between the modelled and observed band ratio appears to superimpose a broad trend throughout the polar night. At sunset, results for the PSR target are similar to the NPSR target, but increasing $\mathfrak{R}_{8 / 9}-\mathfrak{R}_{8 / 9 \text { model }}$ reaches a maximum in mid-night,
02:00 (Fig. 10), then declines towards sunrise. This implies that a reltive reduction in apparent ch. 9 emissivity compared to ch. 8 (or increase in ch. 8 relative to ch. 9) occurs during the night for the PSR target only. Importantly, this time period is when the PSR target is at its coldest (Fig. 3), with mean temperatures dropping below $\sim 40 \mathrm{~K}$. This temperature regime is where we also observe a dramatic increase in $\mathfrak{R}_{8 / 9}-\mathfrak{R}_{8 / 9 \text { model }}$ (Fig. 6). We surmise therefore that this diurnal feature could simply be a further manifestation of an effective temperature-dependence in apparent emissivity ratio that we observe.

\subsection{Insights from thermal conductivity measurements}

The thermal inertia of lunar regolith causes the amplitude of the diurnal thermal wave to taper rapidly with depth in the top $\sim 10 \mathrm{~cm}$ (Hayne et al., 2017), but at the surface and at sub-mm scales, thermal equilibration would occur very quickly.

On average, channel 9 can sense signal from several hundred microns deeper in PSR regolith than channel 8, and any differences in temperature over that short distance could lead to detection of anisothermality in the vertical direction. However, we do not expect to find significant temperature gradients in lunar regolith between the sensing depths of channels 8 and 9. If such gradients were contributors to the signal we observe then (i) they would need to be significant over the difference in sensing depths between channels 8 and 9, a few hundred microns, and (ii) an explanation would then be required for the absence of any local time dependence of $\mathfrak{R}_{8 / 9}-\mathfrak{R}_{8 / 9}$ model for the NPSR target (Fig. 10), particularly at dawn and dusk, when temperatures can change by $>100 \mathrm{~K}$ over relatively short timescales (Fig. 2) and should invoke steep thermal gradients. The absence of evidence for such a dependence suggests that vertical anisothermality is probably not the cause of the observed differences between PSR and NPSR far-IR band ratios. 

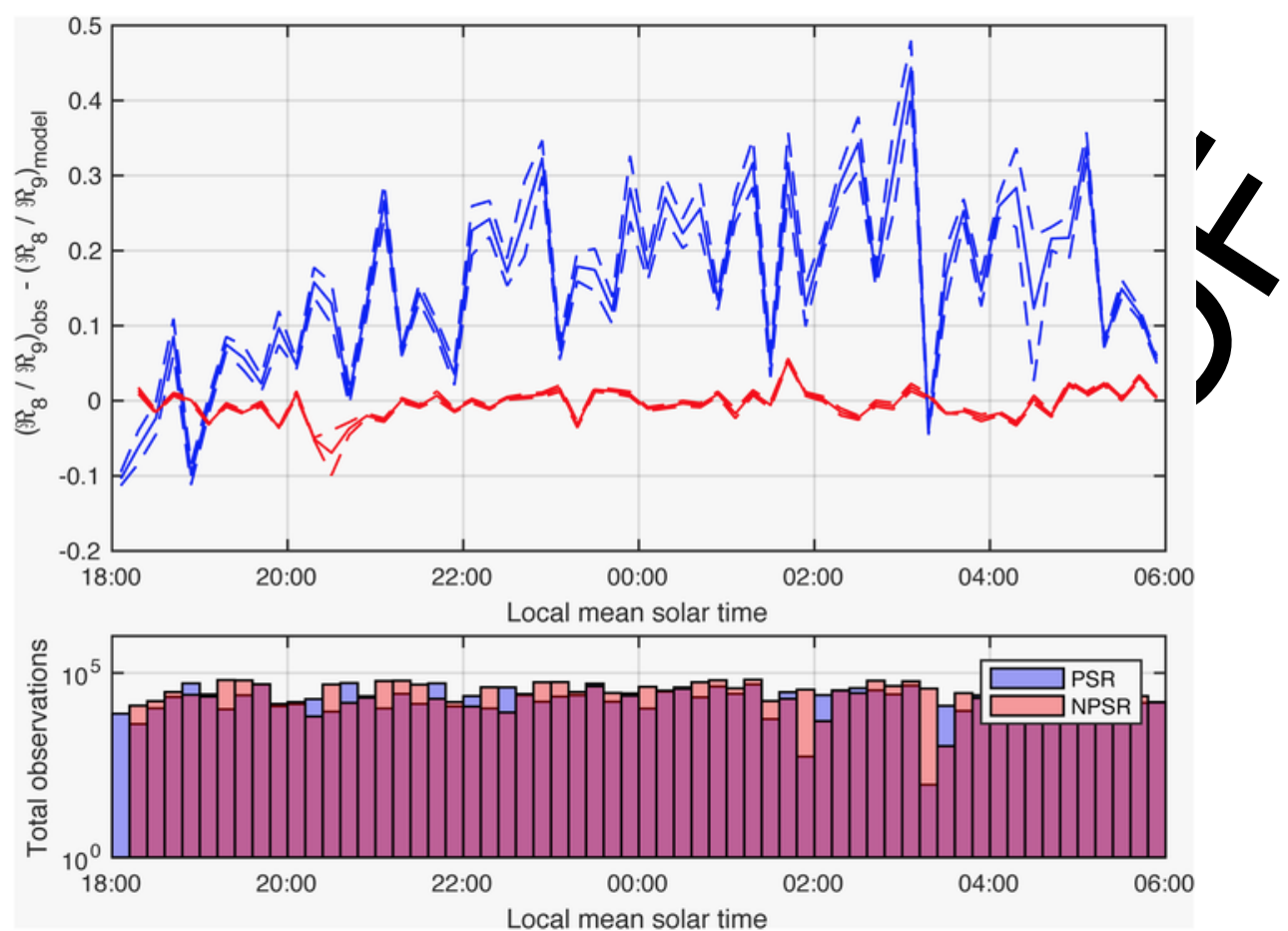

Fig. 10. Top $-\mathfrak{R}_{8 / 9}-\Re_{8 / 9 \text { model }}$ as a function of local mean solar time for each target. Dashed lin indig $\pm 1 \sigma$.Bottom - Total number of observations (log-scale) in both channels 8 and 9 , for each target, plotted as a function of local time (in bins of $12 \mathrm{~min}$ ).

Instead, differences in density between an uppermost and immediately underlying layer could produce differences in thermal conducti ity, and therefore steeper gradients in thermophysical parameters the near surface.

The reduction of thermal conductivity with decreasing densit (e.g Fountain and West, 1970) may be important here to explain the end in the $40-59 \mathrm{~K}$ temperature interval where $\mathfrak{R}_{8 / 9}-\mathfrak{R}_{8 / 9}$ mode non-zero for the PSR target (Fig. 6): The presence of a water frost, or simply lower density of PSR regolith compor of PSR regolith, could be consistent with this.

However, this effect alone would not contribut to t mparent temperature dependence of $\mathfrak{R}_{8 / 9}-\mathfrak{R}_{8 / 9 \text { model be }} 4^{\prime} \mathbb{K}$ (Frg. 6), which we find could be at least partially caused by so FOV doublyshadowed terrain (Fig. 7). Siegler et al. (2016) osen that th variation of thermal conductivity as a function of $t$ nper aro inherently linked to the crystallinity of a given material, an ublished laboratory measurements that indicate that both Ap ro lith and regolith simulant NU-LHT-2M, show thermal conduc ity consistent with amorphous crystalline structure, as mo elled by $V$ pods-Robinson et al. (2019), rather than crystalline materials.

If a thin layer of surface mate rature-d pendenci than the bulk material below, then steeper thermal gradic ts would exist near the surface and result in larger differences bênen ch. 9 brightness temperatures due to their different sensin. depths, and a larger deviation in

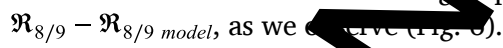

A lack of data in the litere quantifying the effect of ice-regolith mixtures on far-IR emissh proludes our ability to quantify the expected change on $\mathfrak{R}_{8 / 9}$ by urface ice compared to dry regolith. Nonetheless, a thin neer water frost, as indicated by previous studies for the PSR targen (rayne et al., 2015; Fisher et al., 2017) remains consistent with our observations, because differential rates of change in thermal conductivity would occur between an immediate subsurface regolith layer and uppermost volatile layer as a function of temperature.
6. Cont sions

radiance ratio between Diviner channels 8 and 9, when corrected for known instrumental and geometric effects, shows apparent lifferences between normally illuminated and permanently shadowed argets in Amundsen crater. The use of only nighttime data in our analysis leads us to infer this is caused by the two surfaces having different emissivity curves in the far-IR.

The PSR target shows apparent emissivity $\sim 100-400 \mu \mathrm{m}$ (channel 9) lower than expected relative to that between 50 and $100 \mu \mathrm{m}$ (channel 8). The magnitude of the differential increases with decreasing temperature below about $40 \mathrm{~K}$. We do not suspect observation bias to be a factor between 30 and $40 \mathrm{~K}$. For surfaces between $50-70 \mathrm{~K}$, the nonPSR target shows emissivity that is predicted well by a simple Planck radiance model that incorporates the quantum efficiencies of both bandpasses, indicating no differential emissivity in the far-IR.

Via a simple thermal emission model, in which a bimodal temperature distribution is observed by a finite FOV, we demonstrate that it is possible to produce observable anisothermality in the PSR target's anomalous far-IR emissivity curve with the presence of sub-FOV scale cold traps. Such doubly-shadowed terrain could be produced by typical lunar surface roughness in PSRs not more than $\sim 0.45$ areal fraction. However, according to our 2-component thermal emission model, the magnitude of anisothermality that is likely, even for high surface roughnesses, is unable to explain our observations of the far-IR emission curve.

We find inconclusive evidence for emissivity dependence on terrain-relative emission angle, as is theorized for two-layer particulate surfaces, because data coverage is poorest at high emission angles, where the signal would be most obvious. In addition, an apparent broad trend in emissivity with local time for the PSR target may simply be a proxy for the temperature dependence.

Finally, we surmise that the ratio of Diviner far-IR channels 8 and 9, when corrected for all known geometric and instrumental effects, 
may indicate the combined impact on apparent emissivity of two physical properties of PSR surfaces: 1 . The abundance of sub-FOV doublyshadowed terrain harbouring ultra-cool temperatures, and 2. The thermophysical gradient in the uppermost layers of PSR regolith that may be caused by a thin veneer of water ice.

\section{Acknowledgements}

Authors thank the NASA Lunar Reconnaissance Orbiter program and Diviner operations team at NASA Goddard Space Flight Center, NASA/Caltech Jet Propulsion Laboratory, and the University of California Los Angeles for providing data for this research. Special thanks to Marco Delbo for fruitful discussions regarding IR emissivity, and to two reviewers for helpful comments and suggestions that improved the paper. Elliot Sefton-Nash was supported by the European Space Agency. Finally, we remember and celebrate our colleague and friend, Joshua L. Bandfield, who passed during publication of this work. Josh's compassionate life, pursuit of truth, and profound contributions to planetary science remain inspirational.

\section{Appendix A.}

Following Williams et al. (2016), the EFOV is modelled as a Monte Carlo distribution of points in the focal plane. We here describe the change from the instrument/spacecraft-relative coordinate systems in which EFOV points are defined, to 'lunar body-fixed' coordinate system. This must be performed on each point to enable ray-tracing to determine intersection of EFOVs with the lunar digital elevation mod (Teanby, 2009).

Each Monte Carlo point in the EFOV represents a fraction $1 / \eta$ of the total observation. For this study $n_{\text {efov }}=1000$. Each point is d ined as a unit ray with one end at the detector (the start of the barest but that is angularly offset from the boresight at the target lar offsets of the ray from the boresight are in the cross an rections, i.e. parallel and perpendicular, respectively, of each channels' linear detector array. These an treated as spherical coordinates, which allows us look vector into Cartesian coordinates:

$$
\boldsymbol{R}_{D}=x \hat{\boldsymbol{h}}+y \hat{\boldsymbol{k}}+z \hat{\boldsymbol{l}}
$$

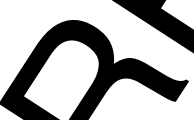

where the unit basis vectors of each component in the detector reference frame, $\boldsymbol{D}$ :

$$
D=\{\hat{\boldsymbol{h}}, \hat{\boldsymbol{k}}, \hat{\boldsymbol{l}}\}
$$

In this right-handed frame, $f$ is the bo pendicular to the direction th detector arrays, and $\hat{l}$ is aligned with the linear detector rays (see Paige et al., 2010b for details). During nominal narion of spacecraft travel, i.e. the in-trac direction, while $\hat{l}$ is the cross-track direction. We must compute in section of each EFOV point with the terrain model, which is storo in the body-fixed lunar reference frame, $\boldsymbol{B}$. Due to the fact $\boldsymbol{B}$ is th reference frame of the digital elevation model (DEM) that we convert EFOV rays into, its component vectors $\{\hat{\boldsymbol{p}}, \hat{\boldsymbol{q}}, \hat{\boldsymbol{r}}\}$ form a $3 \times 3$ identity matrix:

$$
\boldsymbol{B}=\{\hat{\boldsymbol{p}}, \widehat{\boldsymbol{q}}, \widehat{\boldsymbol{r}}\}=\begin{array}{lll}
1 & 0 & 0 \\
0 & 1 & 0 \\
0 & 0 & 1
\end{array}
$$

We construct a change of basis matrix, $\boldsymbol{P}_{\boldsymbol{B} \leftarrow \boldsymbol{D}}$, to convert coordinates from $\boldsymbol{D}$ to $\boldsymbol{B}$, which reduces simply to the components of $\boldsymbol{D}$ :

$$
\begin{array}{rcccccc}
\hat{\boldsymbol{h}} \cdot \hat{\boldsymbol{p}} & \hat{\boldsymbol{h}} \cdot \hat{\boldsymbol{q}} & \hat{\boldsymbol{h}} \cdot \hat{\boldsymbol{r}} & h_{1} & h_{2} & h_{3} \\
\boldsymbol{P}_{B \leftarrow D}=\hat{\boldsymbol{k}} \hat{\boldsymbol{p}} & \hat{\boldsymbol{k}} \cdot \hat{\boldsymbol{q}} & \hat{\boldsymbol{k}} \cdot \hat{\boldsymbol{r}}=k_{1} & k_{2} & k_{3} \\
\hat{\boldsymbol{l}} \hat{\boldsymbol{p}} & \hat{\boldsymbol{l}} \cdot \hat{\boldsymbol{q}} & \hat{\boldsymbol{l}} \cdot \hat{\boldsymbol{r}} & l_{1} & l_{2} & l_{3}
\end{array}
$$

Each EFOV ray is then converted rom the etector frame to the lunar body-fixed frame via multiplica on with e change of basis matrix:

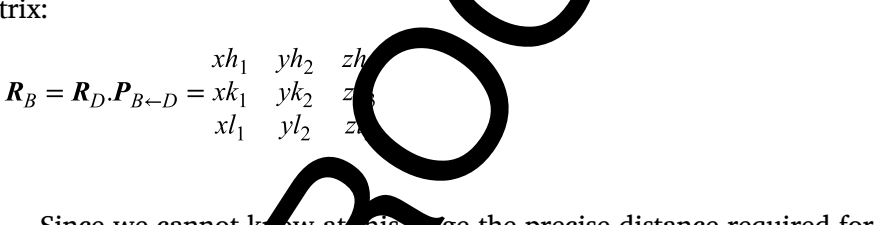

Since we cannot k b at nis suge the precise distance required for intersection of the EFO with the moon's surface, we extend $\boldsymbol{R}_{B}$ by multiplying it by factor, $s$, such that $\left|s \boldsymbol{R}_{B}\right|$ is never too short to interse w with he DEM when an intersection would occur for a ray of infinite

\section{References}

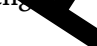

Bandfie J.L., Hayn roughnes derived from LRO Diviner Radiometer observations. Icarus 248 , 7-372. os: Aoi.org/10.1016/j.icarus.2014.11.009.

O., Bada, J, 2002. Extraterrestrial organic compounds in meteorites. Surv. Geophy 23, 411-467. https://doi.org/10.1023/A:1020139302770.

ar J.M. Migliorini, A., Barucci, A., Segura, M., 2006. Constraining the surface Saturn's icy moons, using Cassini/CIRS emissivity spectra. Icarus 187

ph of Saturn's icy moons, using Cassini/CIRS em

aprete, A., Schultz, P., Heldmann, J., Wooden, D., Shirley, M., Ennico, K., Hermalyn, Marshall, W., Ricco, A., Elphic, R.C., Goldstein, D., Summy, D., Bart, G.D., AsPug, E., Korycansky, D., Landis, D., Sollitt, L., 2010. Detection of water in the LCROSS ejecta plume. Science 330, 463-468. https://doi.org/10.1126/science. 1186986.

ottin, H., Gazeau, M.C., Raulin, F., 1999. Cometary organic chemistry: a review from observations, numerical and experimental simulations. Planet. Space Sci. 47, 1141-1162. https://doi.org/10.1016/S0032-0633(99)00024-0.

Delaunay, B., 1934. Sur la sphère vide. vol. 7, Otdelenie Matematicheskikh i. Estestvennykh Nauk, 793-800.

Ehrenfreund, P., Charnley, S.B., 2000. Organic molecules in the interstellar medium, comets, and meteorites: a voyage from dark clouds to the early Earth. Annu. Rev. Astron. Astrophys. 38, 427-483. https://doi.org/10.1146/annurev.astro.38.1.427.

Fisher, E.A., Lucey, P.G., Lemelin, M., Greenhagen, B.T., Siegler, M.A., Mazarico, E., Aharonson, O., Williams, J.-P., Hayne, P.O., Neumann, G.A., Paige, D.A., Smith, D.E., Zuber, M.T., 2017. Evidence for surface water ice in the lunar polar regions using reflectance measurements from the Lunar Orbiter Laser Altimeter and temperature measurements from the Diviner Lunar Radiometer Experiment. Icarus 292, 74-85. https://doi.org/10.1016/j.icarus.2017.03.023.

Fountain, J.A., West, E.A., 1970. Thermal conductivity of particulate basalt as a function of density in simulated lunar and Martian environments. J. Geophys. Res. 75 (20), 4063-4069.

Gladstone, G.R., Stern, S.A., Retherford, K.D., Black, R.K., Slater, D.C., Davis, M.W., Versteeg, M.H., Persson, K.B., Parker, J.W., Kaufmann, D.E., Egan, A.F., Greathouse, T.K., Feldmann, P.D., Hurley, D., Pryor, W.R., Hendrix, A.R., 2010. LAMP: the lyman alpha mapping project on NASA's lunar reconnaissance orbiter mission. Space Sci. Rev. 150 (1-4), 161-181. https://doi.org/10.1007/s11214-009-9578-6.

Hapke, B., 2012. Theory of Reflectance and Emittance Spectroscopy. Cambridge University Press. ISBN: 978-0-521-88349-8.

Hayne, P.O., Hendrix, A., Sefton-Nash, E., Siegler, M.A., Lucey, P.G., Retherford, K.D. Williams, J.-P., Greenhagen, B.T., Paige, D.A., 2015. Evidence for exposed water ice in the moon's south polar regions from lunar reconnaissance orbiter ultra-violet albedo and temperature measurements. Icarus 255, 58-69. https://doi.org/10.1016/ j.icarus.2015.03.032.

Hayne, P.O., Ingersoll, A.P., Paige, D.A., Aharonson, O., Alkalai, L., Byrne, S., Chen, Y., Cohen, B., Colaprete, A., Combe, J.-P., Edwards, C., Ehlmann, B., Feldman, W., Foote, E., Greenhagen, B.T., Hendrix, A., Hermalyn, B., Liu, Y., Lucey, P.G., Malphrus, B., McClanahan, T.P., McCleese, D.J., McCord, T.B., Neish, C., Neumann, G., Nuno, R., Pilorget, C., Poston, M., Sanders, G., Schorghofer, N., Sellar, R.G., Siegler, M., Staehle, R., 2014. New Approaches to Lunar Ice Detection and Mapping. Keck Institute for Space Studies.

Hayne, P.O., Bandfield, J.L., Siegler, M.A., Vasavada, A.R., Ghent, R.R., Williams, J.-P., Greenhagen, B.T., Aharonson, O., Elder, C.M., Lucey, P.G., Paige, D.A., 2017. Global regolith thermophysical properties of the Moon from the Diviner Lunar Radiometer Experiment. Journal of Geophysical Research: Planets 122, 2371-2400. https://doi. org/10.1002/2017JE005387. 
Li, S., Lucey, P.G., Milliken, R.E., Hayne, P.O., Fisher, E., Williams, J.-P., Hurley, D.M., Elphic, R.C., 2018. Direct evidence of surface exposed water ice in the lunar polar regions. Proc. Natl. Acad. Sci. U. S. A. 115 (36), 8907-8912. https://doi.org/10.1073/ pnas. 1802345115

Mazarico, E., Neumann, G.A., Smith, D.E., Zuber, M.T., Torrence, M.H., 2011. Illumination conditions of the lunar polar regions using LOLA topography. Icarus 211, 1066-1081. https://doi.org/10.1016/j.icarus.2010.10.030.

Moore, M.H., Hudson, R.L., 1992. Far-infrared spectral studies of phase changes in water ice induced by proton irradiation. Astrophys. J. 401, 353-360.

Paige, D.A., Foote, M.C., Greenhagen, B.T., Schofield, J.T., Calcutt, S.B., Vasavada, A.R., Preston, D.J., Taylor, F.W., Allen, C.C., Snook, K.J., Jakosky, B.M., Murray, B.C., Soderblom, L.A., Jau, B., Loring, S., Bulharowski, J., Bowles, N.E., Thomas, I.R., Sullivan, M.T., Avis, C., De Jong, E.M., Hartford, W., McCleese, D.J., 2010. The lunar reconnaissance orbiter diviner lunar radiometer experiment. Space Sci. Rev.https:// doi.org/10.1007/s11214-0 09-9529-2.

Paige, D.A., Siegler, M.A., Zhang, J.A., Hayne, P.O., Foote, E.J., Bennett, K.A., Vasavada, A.R., Greenhagen, B.T., Schofield, J.T., McCleese, D.J., Foote, M.C., DeJong, E., Bills, B.G., Hartford, W., Murray, B.C., Allen, C.C., Snook, K., Soderblom, L.A., Calcutt, S., Taylor, F.W., Bowles, N.E., Bandfield, J.L., Elphic, R., Ghent, R., Glotch, T.D., Wyatt, M.B., Lucey, P.G., 2010. Diviner Lunar Radiometer observations of cold traps in the Moon's south polar region. Science 330 (6003), 479-482. https://doi.org/10.1126/ science.1187726.

Rubanenko, L., Aharonson, O., 2017. Stability of ice on the Moon with rough topography. Icarus 296, 99-109. https://doi.org/10.1016/j.icarus.2017.05.028.
Schultz, P.H., Hermalyn, B., Colaprete, A., Ennico, K., Shirley, M., Marshall, W.S., 2010. The LCROSS cratering experiment. Science 330 (6003), 468-472. https://doi.org/10. $1126 /$ science.1187454.

Sefton-Nash, E., Williams, J.-P., Greenhagen, B.T., Aye, K.-M., Paige, D.A., 2017. Diviner lunar radiometer gridded brightness temperatures fr 7 geodesic binning of modeled fields of view. Icarus 298, 98-110. https://doi.org/ .1016/j.icarus.2017.04.007.

Seidelmann, P.K., Abalakin, V.K., Bursa, M., Davie M.E. Bergh, C., Lieske, J.H., Oberst, J., Simon, J.L., Standish, E.M., Stooke, P. As, P.C., 2002. Report of the IAU/IAG working group on cartographic coordinates rotational elements of the planets and satellites: 2000. Celest. Mech.

Siegler, M., Zhong, F., Woods-Robinson, R., P e, D.A., 6 . Low temperature $(<100 \mathrm{~K})$ regolith thermal conductivity - prelimin laboratory ata. In: American Geophysical Union, Fall General Assembly 2016, A ract P24A 5 .

Smith, D., 2017. Lunar Orbiter Laser Altimeter tary Data System.

Task Group on Organic Environment In the Sola ystem, 2007. Exploring Organic Environments in the Solar System. N

Teanby, N.A., 2006. Intersection beth vn spacec es Press, Washington, D. C.

Woods-Robinson, R., Siegler, A., ioe, D.A., . A Model for the Thermophysical Properties of Lunar Regolith Low T unes, Journal of Geophysical Research: Planets, 2019, Accepted manus h ps://doi.org/10.1029/2019JE005955.().

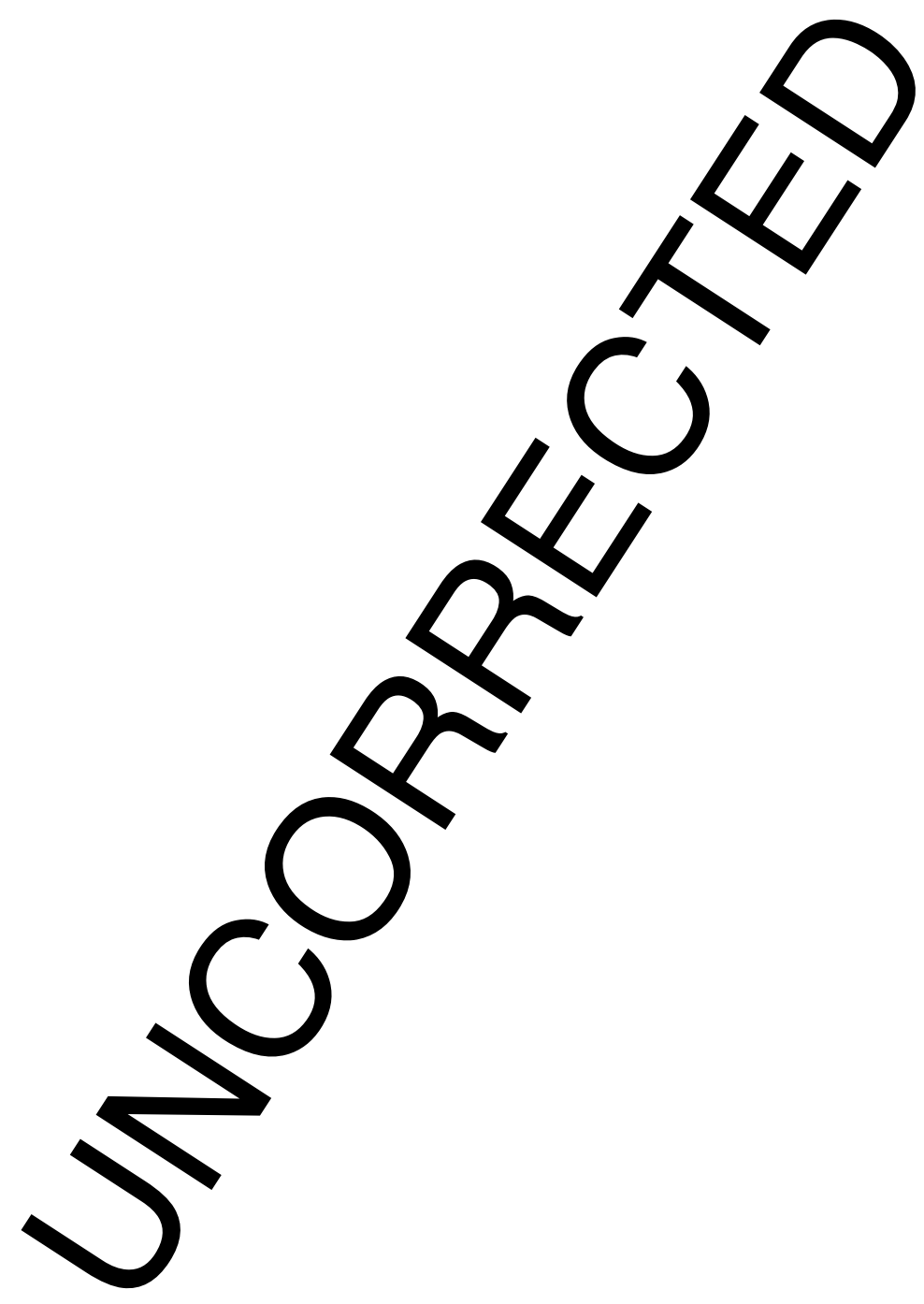

